\title{
RADIATION MODELING WITH DIRECT SIMULATION MONTE CARLO
}

\author{
Ann B. Carlson* \\ NASA Langley Research Center \\ H. A. Hassan** \\ North Carolina State University
}

\begin{abstract}
Improvements in the modeling of radiation in low density shock waves with direct simulation Monte Carlo (DSMC) are the subject of this study. A new scheme to determine the relaxation collision numbers for excitation of electronic states is proposed. This scheme attempts to move the DSMC programs toward a more detailed modeling of the physics and more reliance on available rate data. The new method is compared with the current modeling technique and both techniques are compared with available experimental data. The differences in the results are evaluated. The test case is based on experimental measurements from the AVCO-Everett Research Laboratory electric arc-driven shock tube of a normal shock wave in air at $10 \mathrm{~km} / \mathrm{s}$ and .1 Torr. The new method agrees with the available data as well as the results from the earlier scheme and is more easily extrapolated to different flow conditions.
\end{abstract}

\section{Nomenclature}

* Aerospace Engineer, MS 366, Aerothermodynamics Branch, Space Systems Division, NASA-LARC, Hampton, VA 23665, Senior Member AIAA.

** Professor, Department of Mechanical and Aerospace Engineering, North Carolina State University, Raleigh, NC 27695-7910, Associate Fellow AIAA. 


\begin{tabular}{|c|c|}
\hline$A$ & transition probability \\
\hline$B$ & molecule or atom \\
\hline$g$ & degeneracy \\
\hline$h \nu$ & represents an emitted photon \\
\hline$k$ & Boltzmann constant \\
\hline$K$ & excitation rate coefficient \\
\hline$M$ & molecule, atom or electron \\
\hline$n$ & number density \\
\hline$N$ & number \\
\hline$R$ & collision number \\
\hline$t$ & time \\
\hline$T$ & temperature \\
\hline$x$ & distance coordinate \\
\hline$\epsilon$ & energy of an excited state \\
\hline $\bar{\epsilon}$ & average electronic energy \\
\hline$\theta$ & scattering angle \\
\hline$\lambda$ & wavelength \\
\hline$\nu$ & collision frequency \\
\hline$\sigma$ & cross section \\
\hline$\tau$ & mean radiative lifetime \\
\hline$\phi$ & fraction of state participating \\
\hline
\end{tabular}

\section{Subscripts}

$\begin{array}{ll}a & \text { absorption } \\ e & \text { value for electrons } \\ e q & \text { equilibrium } \\ j, k & \text { value for states } \mathrm{j}, \mathrm{k}\end{array}$


pertaining to particles of type $\mathrm{M}$

$r$

reaction

\section{Introduction}

Recent interest in planetary aerobraking, aeroassisted orbital transfer and hypersonic flight has led to the need to perform analyses in the transitional and high energy flow regimes. At altitudes below the range for free molecule calculations but above the applicability of continuum calculations, the direct simulation Monte Carlo (DSMC) technique is particularly useful. It can, however, be applied more generally. The DSMC method involves the physical simulation of gas particles in a flowfield as they undergo collisions, boundary interactions, and chemical reactions. The success of this method has been in its ability to model highly nonequilibrium flows such as encountered in reentry shock waves. The intent of the current research is to improve the capability of DSMC to model high energy nonequilibrium flows involving radiation.

At reentry velocities in the upper atmosphere, radiation from the shock wave can be a significant portion of the overall heat transfer to the vehicle. Accurate predictions of the nonequilibrium radiation in shocks is required for efficient design of thermal protection systems. Although the ability to model the process of radiation with DSMC has been demonstrated by $\operatorname{Bird}^{1}$, much of the current modeling is very approximate. Verification of the modeling techniques and investigations of alternate techniques are required to improve the level of confidence in the radiation predictions. The present study addresses this goal through investigating the modeling of collisional electronic excitation.

The thermal radiation due to bound-bound transitions between electronic states dominates other forms of radiation under the $10 \mathrm{~km} / \mathrm{s}$ conditions of the current study. One of the main difficulties with modeling this radiation is determining collisional excitation rates for the various excited states. The DSMC method uses a collision number which represents the fraction of collisions between certain species which lead to excitation of electronic states for 
one or both of the particles. Because of the incompleteness of the data available on excitation cross sections, the first selection of these numbers was based on a qualitative knowledge of the magnitudes of the various cross sections. The values were further refined to match available data from the appropriate temperature regime. A new method has been developed which bases these numbers on the available excitation rate data. The rate of production of a species can be expressed in terms of a reaction rate and this, in turn, can be expressed in terms of reaction time, $\tau$. This reaction time, when used in conjunction with the collision frequency, $\nu$, defines a collision number $R=\tau \nu$. Thus, for given excitation rates, it is possible to define excitation numbers which are temperature and species dependent. Such an approach enhances DSMC by reducing the empiricism in the modeling. In addition, it makes it possible to compare with a wider range of alternate calculations and experimental data.

A sample problem of a $10 \mathrm{~km} / \mathrm{s}$ shock wave in air at .1 Torr has been used to evaluate the newly developed method. This test case was chosen because it represents the conditions of a shock tube investigation which was performed by Allen, Rose and Camm in the early 1960 's ${ }^{2}$ and repeated recently by Sharma, Gillespie and Meyer ${ }^{3}$. The calculations are performed with a one-dimensional standing shock wave DSMC program ${ }^{4}$. Differences between the methods are evaluated. Wherever possible, the results are compared with the available measurements.

\section{The DSMC Method}

The direct simulation Monte Carlo (DSMC) method, developed by Professor Graeme $\operatorname{Bird}^{5}$, uses a direct physical simulation approach to solving the flow of low density gases. Because the molecular structure of a gas at low densities must be accounted for in the solution process, the problem lends itself to direct physical description. The current applications of the DSMC method involve the modeling of internal energy, nonequilibrium chemistry, ionizing reactions, and radiation processes. The modeling of thermal radiation 
is discussed in detail in a later section. In most of the other particulars, the DSMC code used in the current predictions is identical to the code which has been well documented by $\operatorname{Bird}^{1}$.

Certain modifications which have been developed by the authors are employed in the calculations presented. The differences pertain to the modeling of ionization and electric field effects. Complete details of these modifications and their effect on the solution can be found in reference 6 . The version of the DSMC code which was used for all the computational results presented in this paper includes the above modifications. Therefore, when results are presented as "Bird's method" or the "new method" these phrases should be interpreted to refer to the differences in radiation modeling only.

\section{Thermal Radiation}

In a shock wave with partial ionization, there is also electronic excitation and accompanying thermal radiation. The radiation from bound-bound transitions between electronic states is known to be significant in $10 \mathrm{~km} / \mathrm{s}$ flows and is the only form of radiation considered in the current study. For more highly energetic flows, the method should be modified to include bound-free and free-free transitions. Because of the large number of radiative states and because a significant amount of the radiation comes from minor species and sparsely populated states, the radiation modeling is handled with a phenomenological modeling technique. Unlike the procedures for the rotational and vibrational energy, in which each molecule is assigned a single state, each excited particle is assigned a distribution over all the available electronic states. This allows the use of a practical number of simulated particles without experiencing the unacceptable statistical scatter which would result if each were assigned a specific excited state and each excitation reaction were modeled directly. Since each particle in a DSMC simulation represents a very large number of real particles, it is not inconsistent with Monte Carlo procedures for a simulated particle to represent a population of real particles with a distribution of excited states. Except as relates to 
the calculation of collisional excitation numbers, the radiation calculations presented herein employ the method of reference 1. A brief description follows.

The sampled electronic energy, $\bar{\epsilon}_{e}$, can be written as an average over the electronic states considered.

$$
\bar{\epsilon}_{e}=\left(\sum \epsilon_{j} N_{j}\right) / N, \quad N=\sum N_{j}
$$

From this it is concluded that a successful event excites the participating simulated particles in accordance with a distribution resulting from the above equation. Therefore,

$$
N_{j} / N=\frac{g_{j} \exp \left(-\epsilon_{j} / k T\right)}{\sum g_{j} \exp \left(-\epsilon_{j} / k T\right)}
$$

The sum is taken over all energies of electronic states below the dissociation or ionization energy. The distribution of states for a particular simulated particle is essentially a Boltzmann distribution, but it depends on the energy of the specific collision which produced the excitation. There is no reason to suppose that the distribution of electronic states in the macroscopic sense is Boltzmann. The list of states considered for molecular and atomic species is given in reference 1 and explained below. This list represents an attempt to include those transitions which are most significant in a $10 \mathrm{~km} / \mathrm{s}$ shock wave. While some of the differences between the experiment and calculations may result from omissions in this set, such investigations are beyond the scope of this study. The information needed to complete the above procedure is the relaxation number for electronic excitation. Determination of the correct relaxation number is the subject of the current investigation.

The molecular band system is the same as that employed by Park $^{7}$ and involves the electronic states of molecular oxygen, neutral and ionized molecular nitrogen, and nitric oxide. The molecular band transitions considered and the mean time to spontaneous emission are listed in Table 1. The actual time to emission in the simulation is assumed to be exponentially distributed about this mean time. One restriction in modeling radiation is that the collision routine time step must be less than the minimum radiative lifetime ${ }^{1}$. As can be seen from Table 1, some of the lifetimes are quite small. Thus, when radiation is 
included, a small simulation time step may be required and long calculation times result.

Table 1. Molecular band system

\begin{tabular}{|c|c|c|c|c|}
\hline Band & States & $\tau(\mathrm{s})$ & $\lambda$ (microns) & $(\mathrm{ev})$ \\
\hline$N_{2}, 1+$ & $3 \rightarrow 2$ & $1.1 \times 10^{-5}$ & 1.06 & 1.17 \\
$N_{2}, 2+$ & $5 \rightarrow 3$ & $2.7 \times 10^{-8}$ & 0.34 & 3.65 \\
$O_{2}, S-R$ & $5 \rightarrow 1$ & $8.2 \times 10^{-9}$ & 0.20 & 6.20 \\
$N O, \beta$ & $3 \rightarrow 1$ & $6.7 \times 10^{-7}$ & 0.22 & 5.64 \\
$N O, \gamma$ & $2 \rightarrow 1$ & $1.16 \times 10^{-7}$ & 0.23 & 5.39 \\
$N_{2}^{+}, 1-$ & $3 \rightarrow 1$ & $6.7 \times 10^{-8}$ & 0.39 & 3.18 \\
\hline
\end{tabular}

The states for atomic radiation were combined to form a manageable number of groups ${ }^{1}$. Therefore, the radiative transitions are also grouped and are referred to by number rather than spectroscopic code. As a consequence of the grouping, each transition generally involves only a fraction of the states in the upper group. This fraction, $\phi$, is included in the tables of radiative transitions. These tables are Tables 2 and 3 for atomic oxygen and nitrogen, respectively.

Table 2. Radiative transitions for $O$

\begin{tabular}{|c|c|c|c|c|c|}
\hline Number & Transition & $\phi$ & $\tau(\mathrm{s})$ & $\lambda$ (microns) & $(\mathrm{ev})$ \\
\hline 1 & $5 \rightarrow 4$ & 0.6 & $3.3 \times 10^{-8}$ & 0.83 & 1.49 \\
2 & $6 \rightarrow 1$ & 0.1 & $2.5 \times 10^{-8}$ & 0.103 & 12.0 \\
3 & $6 \rightarrow 4$ & 0.01 & $2 . \times 10^{-6}$ & 0.45 & 2.76 \\
4 & $6 \rightarrow 5$ & 0.7 & $2.5 \times 10^{-8}$ & 0.99 & 1.25 \\
5 & $7 \rightarrow 1$ & 0.1 & $0.4 \times 10^{-8}$ & 0.099 & 12.5 \\
6 & $7 \rightarrow 5$ & 0.4 & $1 . \times 10^{-7}$ & 0.64 & 1.94 \\
7 & $8 \rightarrow 5$ & 0.1 & $5 . \times 10^{-7}$ & 0.55 & 2.25 \\
\hline
\end{tabular}


Table 3. Radiative transitions for $N$

\begin{tabular}{|c|c|c|c|c|c|}
\hline Number & Transition & $\phi$ & $\tau(\mathrm{s})$ & $\lambda$ (microns) & $(\mathrm{ev})$ \\
\hline 1 & $4 \rightarrow 1$ & 0.5 & $0.5 \times 10^{-8}$ & 0.117 & 10.6 \\
2 & $4 \rightarrow 2$ & 0.25 & $0.2 \times 10^{-8}$ & 0.152 & 8.16 \\
3 & $4 \rightarrow 3$ & 0.25 & $0.5 \times 10^{-8}$ & 0.178 & 6.97 \\
4 & $5 \rightarrow 2$ & 0.15 & $0.2 \times 10^{-8}$ & 0.129 & 9.61 \\
5 & $5 \rightarrow 4$ & 0.08 & $6 . \times 10^{-8}$ & 0.907 & 1.37 \\
6 & $6 \rightarrow 2$ & 0.1 & $1 . \times 10^{-8}$ & 0.117 & 10.6 \\
7 & $6 \rightarrow 3$ & 0.1 & $1 . \times 10^{-8}$ & 0.132 & 9.39 \\
8 & $6 \rightarrow 5$ & 0.7 & $5 . \times 10^{-8}$ & 1.19 & 1.04 \\
9 & $7 \rightarrow 4$ & 0.1 & $1 . \times 10^{-6}$ & 0.45 & 2.76 \\
10 & $8 \rightarrow 2$ & 0.005 & $3 . \times 10^{-8}$ & 0.108 & 11.5 \\
11 & $8 \rightarrow 3$ & 0.003 & $5 . \times 10^{-8}$ & 0.121 & 10.2 \\
12 & $8 \rightarrow 4$ & 0.008 & $2.5 \times 10^{-7}$ & 0.38 & 3.26 \\
13 & $8 \rightarrow 5$ & 0.04 & $1 . \times 10^{-7}$ & 0.65 & 1.91 \\
\hline
\end{tabular}

A simple physical model has been used in the calculations to estimate the probable effects of absorption in the flow. The simple model is thought to be adequate for the visible and infrared portions of the spectrum because the gas is essentially transparent to the radiation over most of this wavelength range. The transparency assumption looses validity in the ultraviolet and the calculations in this wavelength range may be degraded as a result. If the number density of absorbing particles is $n_{a}$ in a cell of width $\Delta x$, the probability of absorption of a photon moving at an angle of inclination $\theta$ to the axis in that cell is

$$
\Delta x n_{a} \sigma_{a} / \cos \theta
$$

In this simple model, $\sigma_{a}$ is assumed to be constant. Each time a radiation event occurs, the angle $\theta$ is chosen such that all directions are equally possible and the trajectory of the photon is followed until it is absorbed in the flow, hits the surface, or exits from the flow. For the present calculation, the energy of the absorbed radiation is not put back into the flow. The effect of neglecting this energy will be small because the amount of absorption is small. However, absorption in the ultraviolet portion of the spectrum should be the subject 
of further investigation.

\section{Collisional Excitation Numbers}

There are not much available data on the excitation cross sections of the species involved in a real air model. Therefore, the determination of the ratio of collision cross section to excitation cross section, which yields the collision number, is not an easy task. Separate collision numbers are needed for collisions of each species with neutrals, ions, and electrons. A primarily qualitative method was adopted by Bird to demonstrate the capability of DSMC to predict nonequilibrium radiation. This method is outlined below.

The electron-ion and electron-neutral elastic cross sections are of the order of $10^{-15}$ $\mathrm{cm}^{2}$ and the data that are available suggest that electron impact excitation cross sections are on the order of $10^{-16} \mathrm{~cm}^{2}$. This indicates a relaxation collision number of about 10 . Bird argued that, because a successful event was assumed to yield a distribution of states instead of a single state, a reduction in the cross sections (or an increase in the collision number) was necessary. Table 4 shows the relaxation collision numbers which were used for electron and ion impact reactions. The values were increased by a factor of 10 for collisions with neutrals.

Table 4. Collision numbers for excitation, Bird's method

\begin{tabular}{|c|c|}
\hline Species & Number \\
\hline $\mathrm{O}_{2}$ & 100 \\
$\mathrm{~N}_{2}$ & 100 \\
$\mathrm{O}$ & 500 \\
$\mathrm{~N}$ & 1000 \\
$\mathrm{NO}$ & 100 \\
$\mathrm{~N}_{2}^{+}$ & 100 \\
\hline
\end{tabular}

Because the numbers employed in this table are rather approximate, we believe that such a procedure detracts from using DSMC in future radiation calculations. It is also our 
contention that if we have the correct relaxation numbers for the model then there is no need to compensate for replacing a level by a distribution.

\section{The Proposed Method}

The object of the investigation was to provide a method to calculate the relaxation numbers which is based on the existing data and does not introduce empiricism into the procedure. This can be accomplished by using the following procedure which depends on the excitation rate data.

The reactions which are responsible for electronic transitions between lower state, $j$, and upper state, $k$, are

$$
M+B_{j} \longleftrightarrow M+B_{k}
$$

and

$$
B_{k} \longleftrightarrow B_{j}+h \nu
$$

The largest rates in these reactions are for collisions with charged particles. However, for a slightly ionized gas, neutral particle collisions are also important. The production rate of species $B_{j}$ with number density $n_{j}$ is

$$
\begin{gathered}
d n_{j} / d t=\sum_{k} K_{M}(k, j) n_{k} n_{M} \\
-\sum_{k} K_{M}(j, k) n_{j} n_{M}+\sum_{k} A(k, j) n_{k}-\sum_{k} A(j, k) n_{j}
\end{gathered}
$$

Multiplying by the energy of level $j, \epsilon_{j}$, and summing it is possible to apply the standard Landau and Teller $^{8}$ theory to express the result in terms of a local relaxation time, $\tau$.

$$
\begin{gathered}
n d \bar{\epsilon}_{e} / d t=d / d t\left(\sum \epsilon_{j} n_{j}\right)= \\
n_{M} \sum_{j} \epsilon_{j} \sum_{k} K_{M}(k, j) n_{k}-n_{M} \sum_{j} \epsilon_{j} n_{j} \sum_{k} K_{M}(j, k)+\sum_{j} \epsilon_{j} \sum_{k} A(k, j) n_{k}-\sum_{j} \epsilon_{j} n_{j} \sum_{k} A(j, k) \\
\equiv n\left(\bar{\epsilon}_{e q}-\bar{\epsilon}_{j}\right) / \tau
\end{gathered}
$$


If the excitation and deexcitation terms on the left hand side of equation 7 are assumed to be of the same order of magnitude as the terms representing the departure of the energy from equilibrium on the right hand side, $1 / \tau$ can be estimated as

$$
1 / \tau \simeq \frac{\sum \epsilon_{j} n_{j}\left(K_{M}(j) n_{M}+A(j)\right)}{\sum \epsilon_{j} n_{j}}
$$

when

$$
\begin{aligned}
K_{M}(j) & =\sum_{k} K_{M}(j, k) \\
A(j) & =\sum_{k} A(j, k)
\end{aligned}
$$

The above equation, while only an estimate for the appropriate relaxation time, should be an improvement over the previous method because it does incorporate the available excitation rate data and flowfield property variations.

The fraction of collisions which lead to excitation is equal to $1 / \tau \nu$ (or the collision number for excitation is $\tau \nu$ ). The needed rate coefficients for charged particle impact are available from Park's NEQAIR radiation code $^{9}$ with the exception of those for molecular oxygen and the highest excited level of molecular nitrogen. Those rates were taken from Slinker and $\mathrm{Ali}^{10}$.

According to Park, ${ }^{7}$ the cross sections for the collisions of neutral heavy particles are smaller, approximately by the ratio of the two masses, than those of electron collisions. In this study the collision numbers for neutral particles have been determined from this relationship, although further investigation of the accuracy of this assumption is recommended.

In order to compare with Table 4, sample collision numbers for the test case are given in Table 5. These collision numbers represent the values obtained when the average temperature and species densities from the significantly radiating portion of the flowfield were used to evaluate the rate coefficient and transition probabilities of equations $6-9$.

Table 5. Collision numbers for excitation, new method 


\begin{tabular}{|c|c|}
\hline Species & Number \\
\hline$O_{2}$ & 34 \\
$N_{2}$ & 4 \\
$O$ & 1 \\
$N$ & 1 \\
$N O$ & 13 \\
$N_{2}^{+}$ & 1 \\
\hline
\end{tabular}

While much smaller than the collision numbers used by Bird, these values are in the expected range for the neutral particles. The numbers for atoms are smaller than expected but within the range of possibility. This may indicate a need for some adjustments to the procedure. There is a problem with the value for excitation of $N_{2}^{+}$which has very high excitation rate coefficients compared with the other species. In this case the value was generally less than 1 . The program requires the collision number to be 1 or greater. Therefore the value for $N_{2}^{+}$is set to 1 , resulting in a model of the excitation of $N_{2}^{+}$which is collision limited. Recognizing that the entire procedure is approximate in nature and that $N_{2}^{+}$is known to be a strong radiator which is only slightly affected by nonequilibrium phenomena $^{11}$, this adjustment is not expected to significantly degrade the results.

The Larsen Borgnakke approach which is used for the partitioning of electronic energy is also the approach used for the partitioning of the rotational and vibrational internal energy. This consistency of approach for the partitioning of internal energy is one of the advantages of the DSMC method of modeling nonequilibrium flows. The approach depends on the correct calculation of relaxation numbers for determining the percentage of collisions which result in the redistribution of internal energy. If these relaxation numbers are determined correctly, then it will not matter whether the energy is assigned to a specific internal state, as with rotation and vibration, or to a distribution of states, as with electronic excitation. Since radiation is determined by the electronic energy, then as long as the average, $\bar{\epsilon}$, is computed correctly, the electronic energy of the system will remain the same whether it is assigned to one state or a distribution of states. As a result, when a large sample is 
considered, the results should be independent of the manner in which $\bar{\epsilon}$ is assigned. Thus, it is not necessary to alter the relaxation numbers to account for assuming a distribution of states, as was done in Bird's procedure.

\section{Results}

Both global radiation values and the spectral distribution are investigated. The recent measurements by Sharma, et al. agree well with the earlier shock tube result. Therefore direct comparison is restricted to the data of reference 2 at this time. For convenience in displaying the results, a shock center is defined as the point at which the density is 6 times the free stream density. This is defined as $x=0$ in the flowfield plots (Figs. 1 and 3 ). Molecules travel from $-x$ to $+x$ on average in the flow.

The global radiation results (Fig. 1) show large differences between the two collision excitation number methods. When Bird's method is used the intensity of radiation near the center of the shock is much lower. The present method predicts larger radiation levels overall, but particularly in the nonequilibrium region.

The general radiation pulse shape from the oscillogram trace during the shock tube experiment is pictured in Fig. 2. The calculated emission is plotted against distance but, since the wave moves $1 \mathrm{~cm}$ in one microsecond, the comparison with the trace is easily made. The intensity scale of the trace was not calibrated, so absolute comparisons cannot be made. However, the peak nonequilibrium intensity appears to be about one order of magnitude greater than the relatively level equilibrium intensity immediately behind the shock. The rise in equilibrium intensity as one moves away from the shock was attributed in the Allen, et al. paper ${ }^{2}$ to the attenuation of the shock velocity as it moves down the tube. It is not relevant to the computations. The new method yields a ratio of nonequilibrium to equilibrium intensity which is more in line with the oscillogram trace than the results of Bird's method.

The translational temperature and electron temperature for the two methods are shown 
in Fig. 3. Peak translational and electron temperatures occur ahead of the shock center, as defined by the density ratio, except in the case of the new method electron temperature. The translational temperature is slightly lower in the present method, but the most noticeable difference is the lower electron temperature in the region just ahead of the shock center. The larger amount of radiation corresponds to proportionately less energy in the other modes, hence the lower temperatures.

\section{Nonequilibrium Radiation}

The experimental results for the radiation intensity versus wavelength in the nonequilibrium (peak radiation) region are compared in Fig. 4 with the results from the two DSMC calculations. The radiant energy per unit volume from each of the transitions listed in Tables 1, 2, and 3 was spread evenly over the wavelength range defined by the midpoints between this transition and the two neighboring transitions. Both methods have reasonable qualitative agreement with the experimental data except for the present method overshoot of radiation at .39 microns. In general the present method tends to overpredict the radiation in the lower wavelength region while Bird's method tends to underpredict the radiation in the longer wavelengths. The overshoot at .39 microns is a result of the large amount of radiation from the $N_{2}^{+},[1-]$ state. As mentioned before, the electronic excitation rates for this ion were very large compared with the rates for the other species and a collision number could not be calculated directly. This uncertainty could be blamed for the calculation discrepancy. However, the ion is known to be a strong radiator and a small overestimation of the concentration of $N_{2}^{+}$at this point in the flow could equally well account for the radiation overshoot. A detailed comparison of the radiation from separate species and transitions is given in Figs. 5, 6, and 7. The contribution to the radiation from the molecular species is consistently larger with the new method. The largest increase in the molecular contribution is the contribution from $N_{2}^{+}$, as was expected from the radiation overshoot at .39 microns evident in Fig. 4. In the region below .2 microns the present method shows 
a significantly larger contribution from $N$ and $O$ atom transitions. While this discrepancy might be attributed to the smaller collision numbers for atomic species of the new method, differences in chemistry or electron energy could also be a factor. Unfortunately, not only is the mechanism for this unclear, but no data exist to compare with the computations in this region.

\section{Equilibrium Radiation}

The spectral distribution of radiation from the near equilibrium region (region of relatively constant radiation) behind the shock is compared to the shock tube data for the two calculations in Fig. 8. Detailed plots of the contributions from the molecular and atomic species are plotted in Figs. 9, 10, and 11. The results are similar to the nonequilibrium region in that the new method predictions tend to be larger than those of Bird's method, although the magnitude of the disagreement is less in the near equilibrium region.

\section{Conclusions}

In an effort to improve the modeling of nonequilibrium radiation with DSMC, a new method for determining relaxation collision numbers for electronic excitation has been proposed. Results for the new model are compared with results obtained using the method currently incorporated in Bird's DSMC programs ${ }^{1}$. These results are also compared with data from an electric arc-driven shock tube experiment ${ }^{2}$.

The determination of the relaxation collision numbers for electronic excitation was previously based on a qualitative knowledge of the magnitudes of excitation cross sections compared with collision cross sections. The numbers required some adjustment in order to obtain agreement with experiment. A new method was proposed which bases the determination of these numbers on the available rate data for electronic excitation and values of radiative state lifetimes. It has been shown that the relaxation collision numbers obtained with this method can be used without adjustment to provide equally acceptable radiation 
estimates. With the exception of the contribution to the total radiation from the $\mathrm{N}_{2}^{+}$ion, the results obtained with the new method agree as closely with the experimental values as those obtained with Bird's method. The $\mathrm{N}_{2}^{+}$predictions may be off for a number of reasons. The most probable are errors in collision number due to calculation uncertainties and/or errors in ion concentration due to uncertainties in the nonequilibrium chemistry. Further work is suggested. More detailed modeling of the effect of absorption in the ultraviolet portion of the spectrum is also suggested.

The methods both suffer from the lack of experimental data. These data are required to supply some of the modeling parameters. They are also needed in order to evaluate whether the methods are adequate for future DSMC applications. However, judging from the data that are available, the new modeling technique appears to be a step in the right direction.

\section{Acknowledgement}

This work was supported in part by NASA's Cooperative Agreement NCCI-112, the Hypersonic Aerodynamic Program Grant NAGW-1022 funded jointly by NASA, AFOSR and ONR, and the Mars Mission Research Center, funded by NASA's Grant NAGW-1331. The authors would like to express their appreciation to Professor G. A. Bird for his guidance and advice.

\section{References}

1. G. A. Bird, "Nonequilibrium Radiation During Reentry at $10 \mathrm{~km} / \mathrm{s}$," AIAA Paper $87-1543,1987$.

2. R. A. Allen, P. H. Rose, and J. C. Camm, "Nonequilibrium and Equilibrium Radiation at Super-Satellite Reentry Velocities," Research Report 156, AVCO-Everett Research Laboratory, Everett, Mass, 1962.

3. S. P. Sharma, W. D. Gillespie, and S. A. Meyer, "Shock Front Radiation Measurements in Air," AIAA Paper 91-0573, 1991. 
4. G. A. Bird, "Direct Simulation of Typical AOTV Entry Flows," AIAA Paper 86-1310, 1986.

5. G. A. Bird, Molecular Gas Dynamics, Oxford University Press, London, 1976.

6. A. B. Carlson and H. A. Hassan, "Direct Simulation of Reentry Flows with Ionization," AIAA Paper 90-0144, 1990.

7. C. Park, "Calculation of Nonequilibrium Radiation in the Flight Regimes of Aeroassisted Orbital Transfer Vehicles," Progress in Astronautics and Aeronautics, Vol 96, pp395-418, 1985 .

8. L. Landau and E. Teller, "Zur Theorie der Schalldispersion," Physik Z. Sowjetunion, b. 10 , h. 1, p. 34,1936 .

9. C. Park, "Nonequilibrium Air Radiation (NEQAIR) Program: Users Manual," NASA TM 86707, 1985.

10. S. Slinker and A. W. Ali, "Electron Excitation and Ionization Rate Coefficients for $\mathrm{N}_{2}$, $\mathrm{O}_{2}, \mathrm{NO}, \mathrm{N}$, and $O, "$ NRL Memorandum Report 4756, Naval Research Laboratory, 1982.

11. L. A. Carlson and T. A. Gally, "Nonequilibrium Chemical and Radiation Coupling Phenomena in AOTV Flowfields," AIAA Paper 91-0569, 1991. 


\section{Captions}

Fig. 1 Global radiation through the shock.

Fig. 2 Oscillogram records of radiation from shock tube experiment.

Fig. 3 Temperature through the shock.

Fig. 4 Nonequilibrium spectral radiation intensity vs. wavelength.

Fig. 5 Molecular contributions to total nonequilibrium radiation.

Fig. 6 Contribution of atomic nitrogen to total nonequilibrium radiation.

Fig. 7 Contribution of atomic oxygen to total nonequilibrium radiation.

Fig. 8 Equilibrium spectral radiation intensity vs. wavelength.

Fig. 9 Molecular contributions to total equilibrium radiation.

Fig. 10 Contribution of atomic nitrogen to total equilibrium radiation.

Fig. 11 Contribution of atomic oxygen to total equilibrium radiation. 
Fig. 1

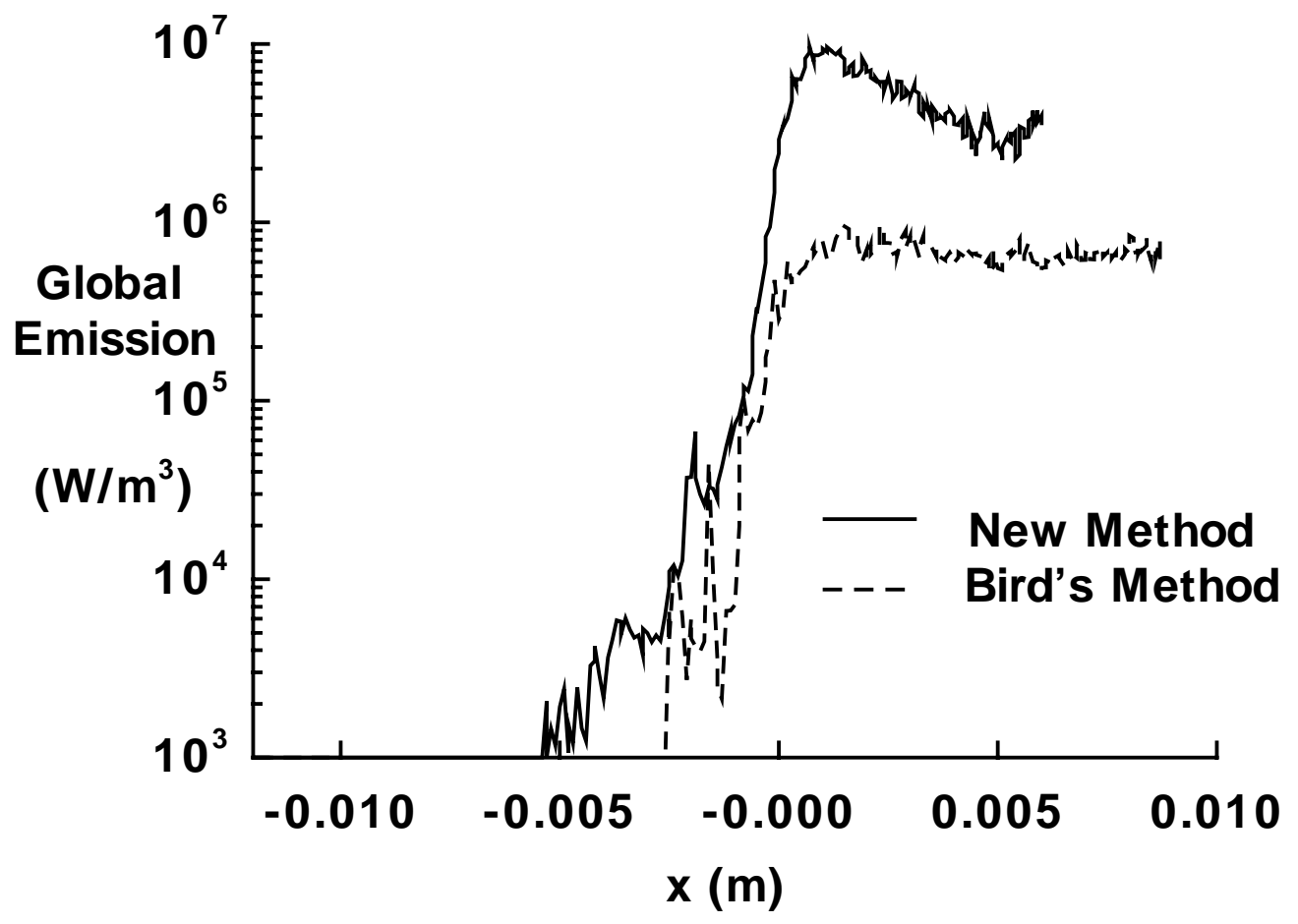


Fig. 2

Not available 
Fig. 3

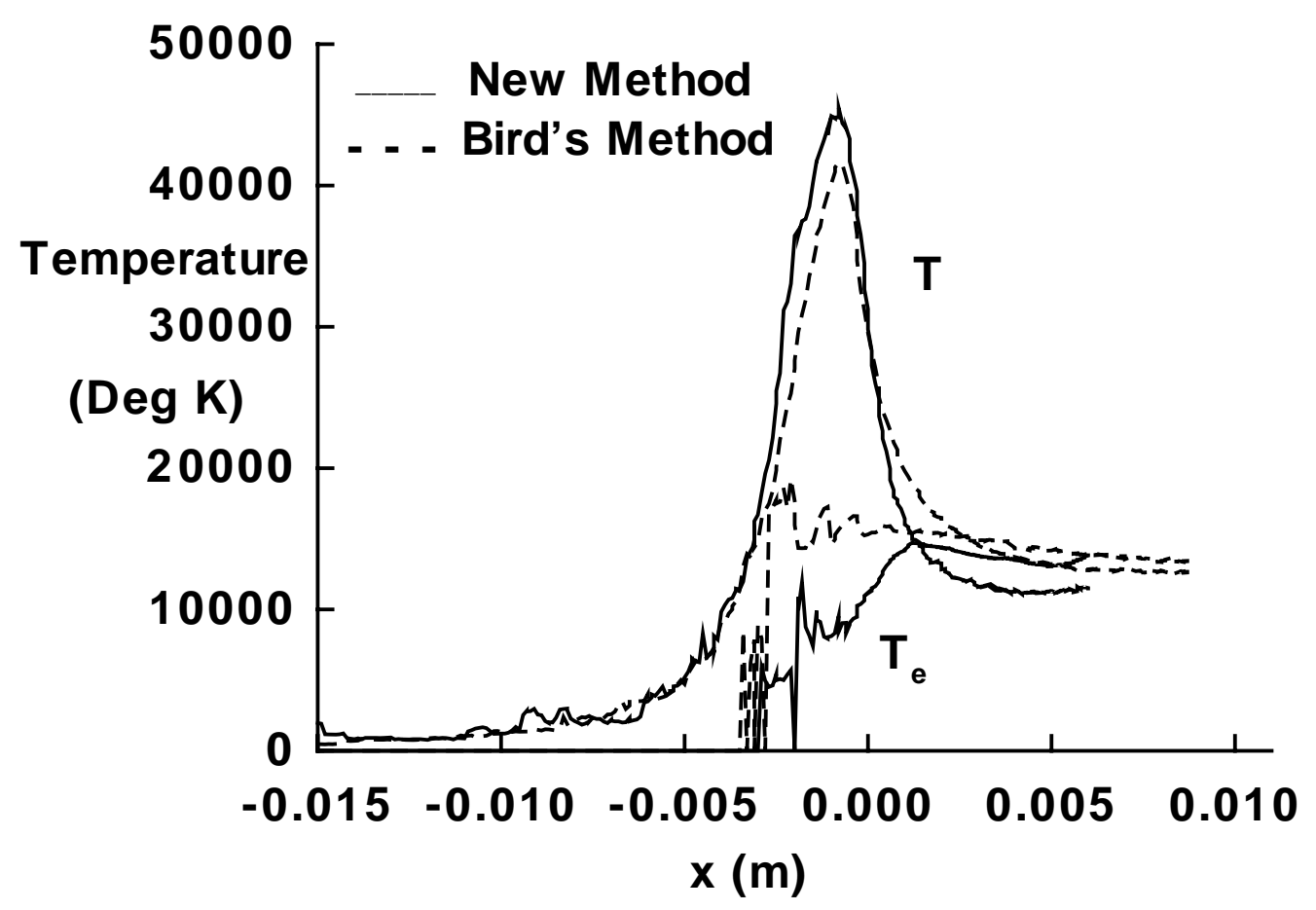


Fig. 4

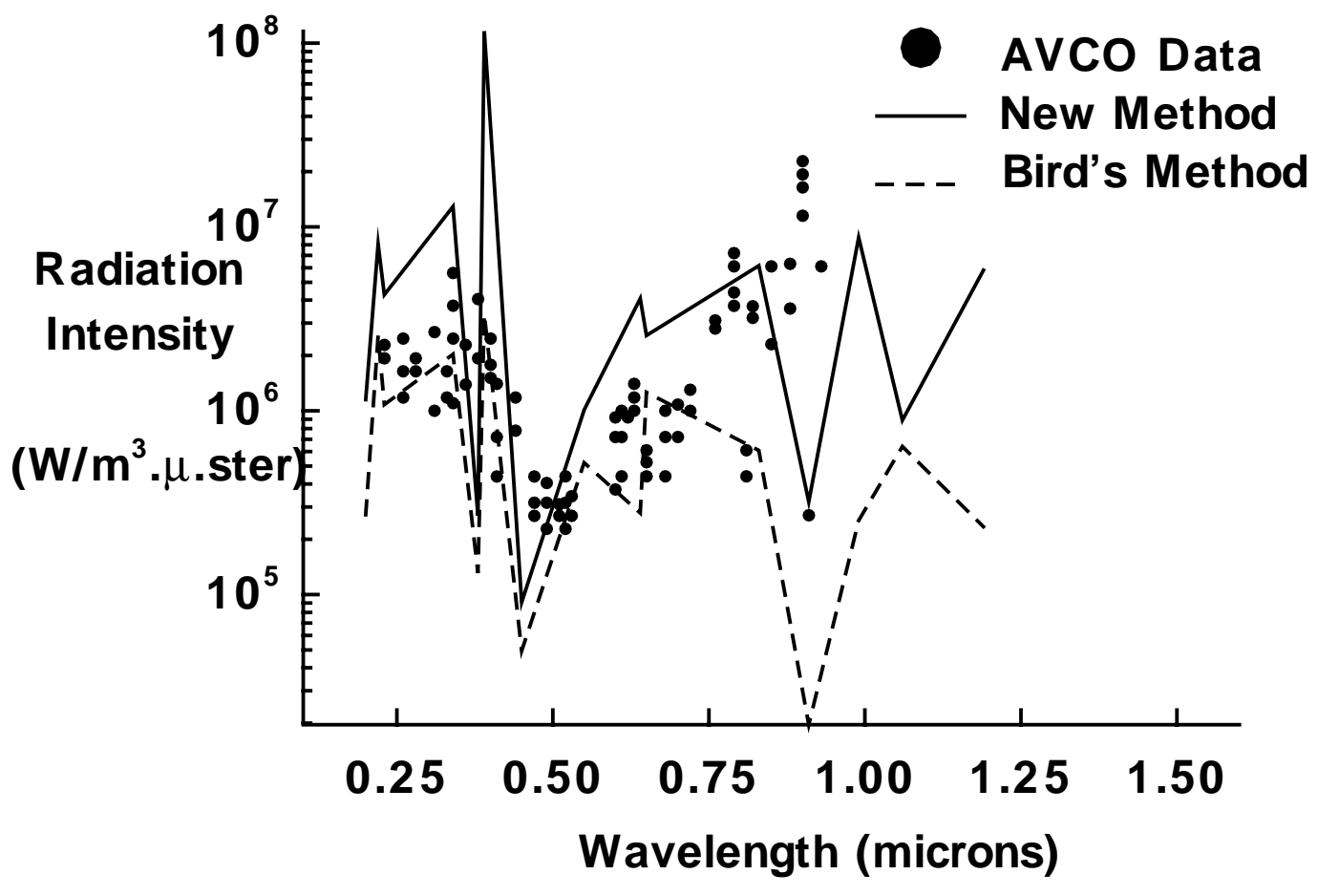


Fig. 5

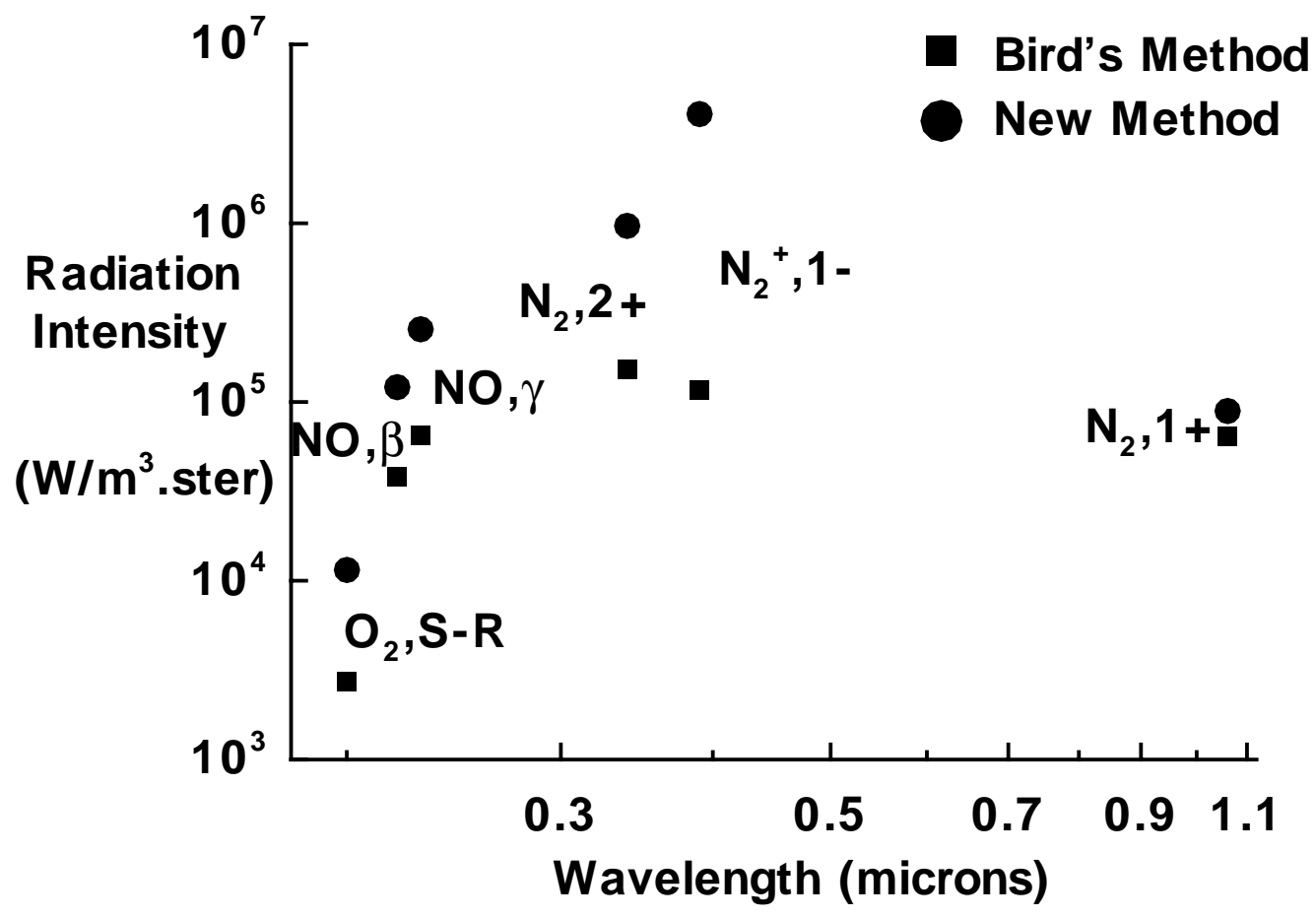


Fig. 6

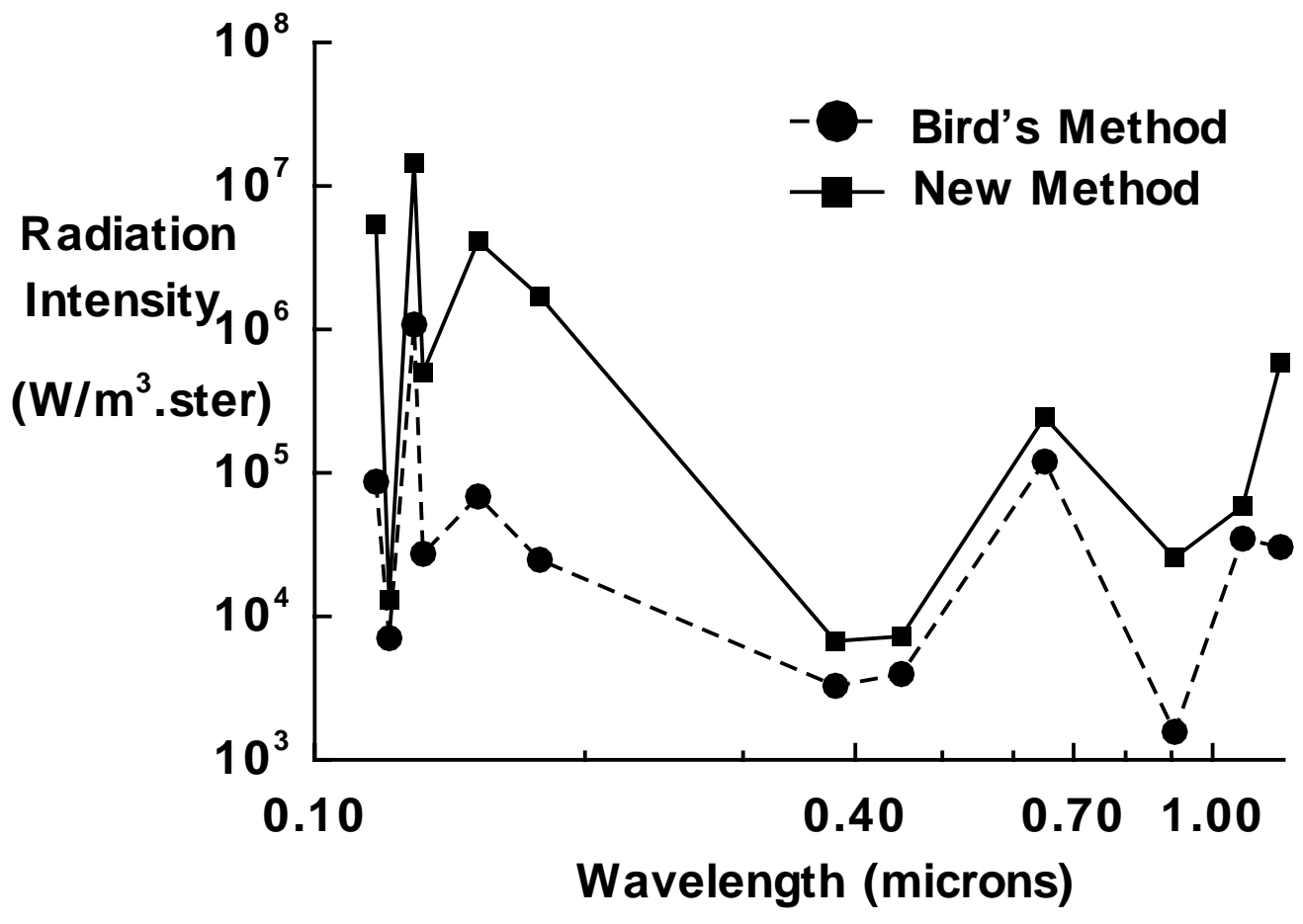


Fig. 7

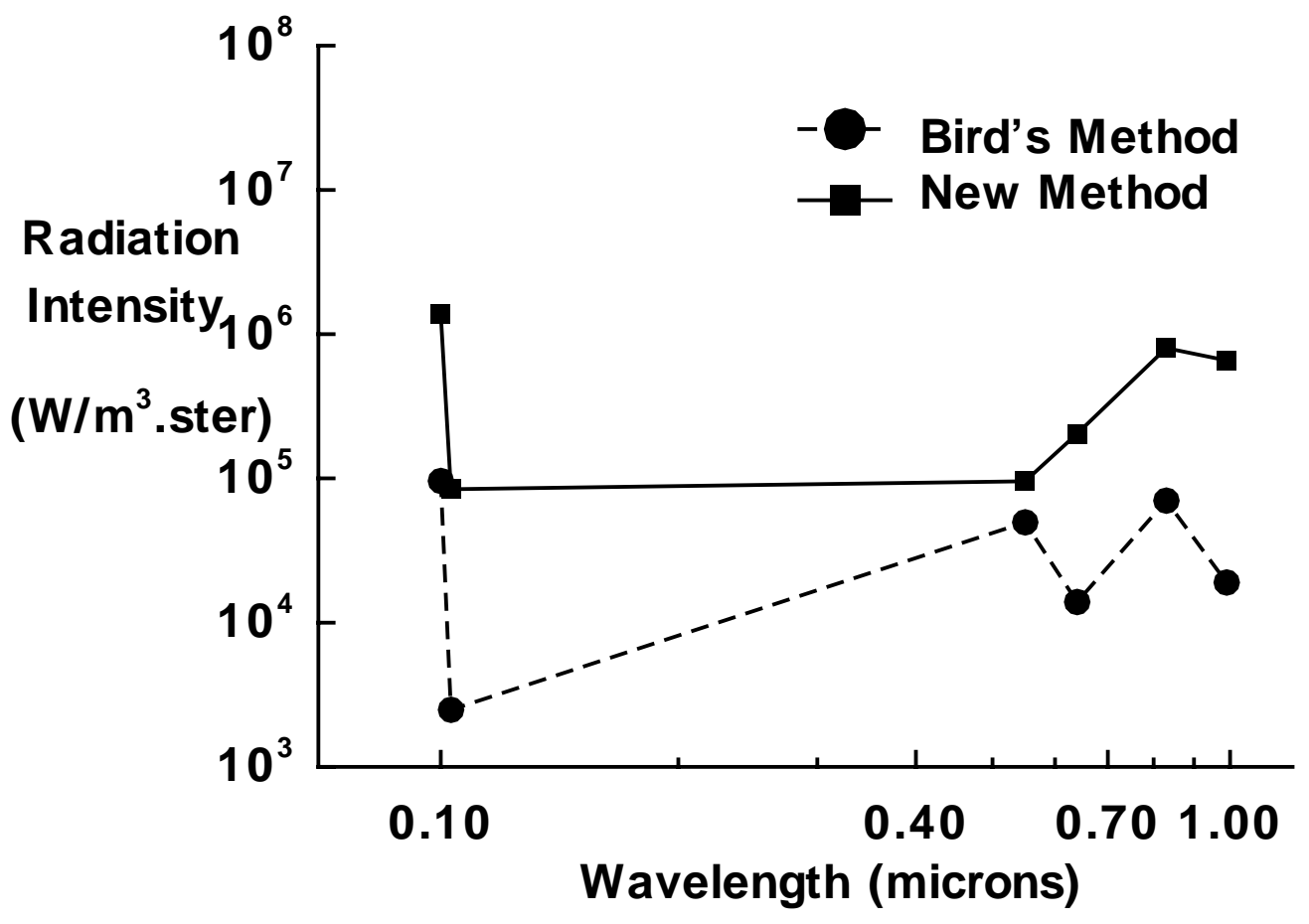


Fig. 8

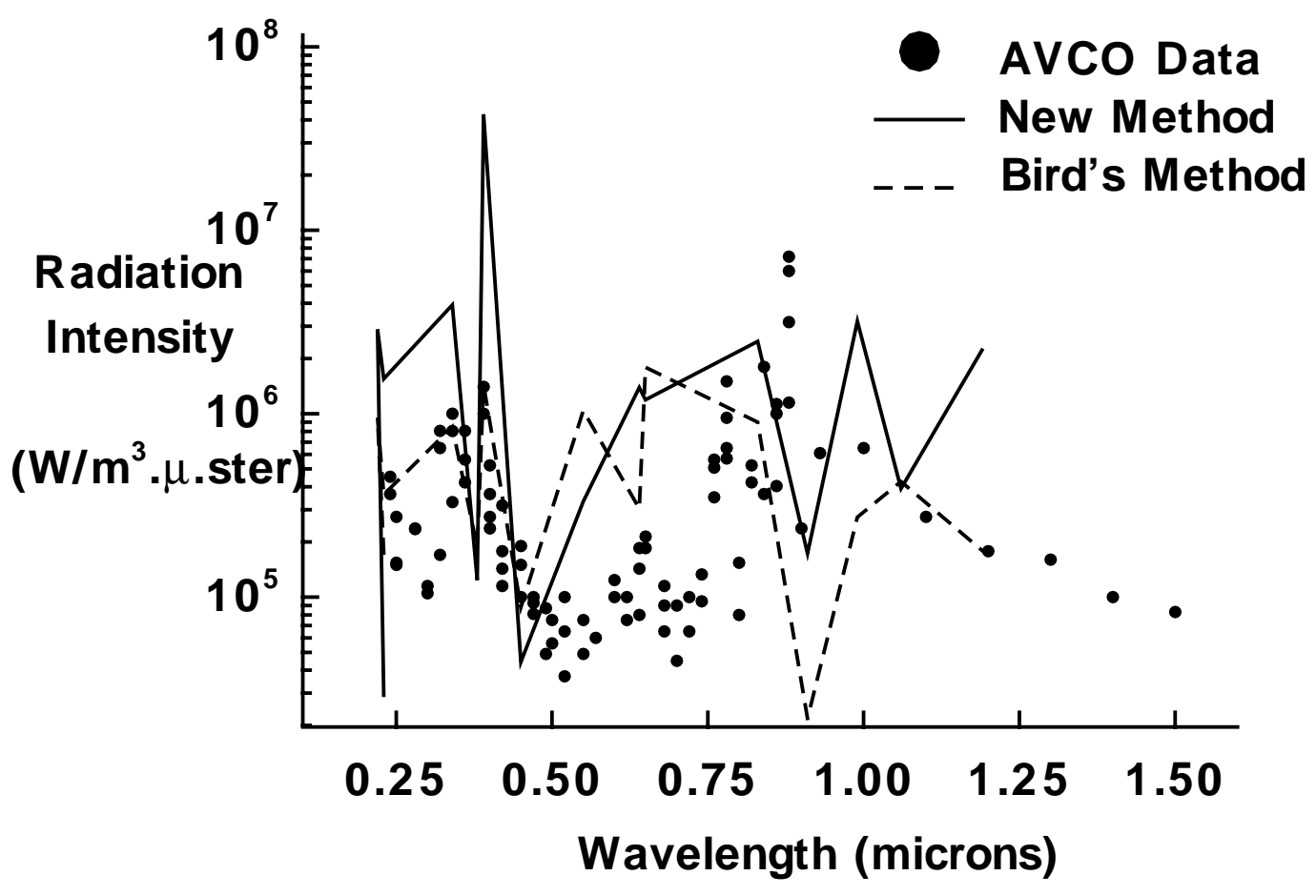


Fig. 9

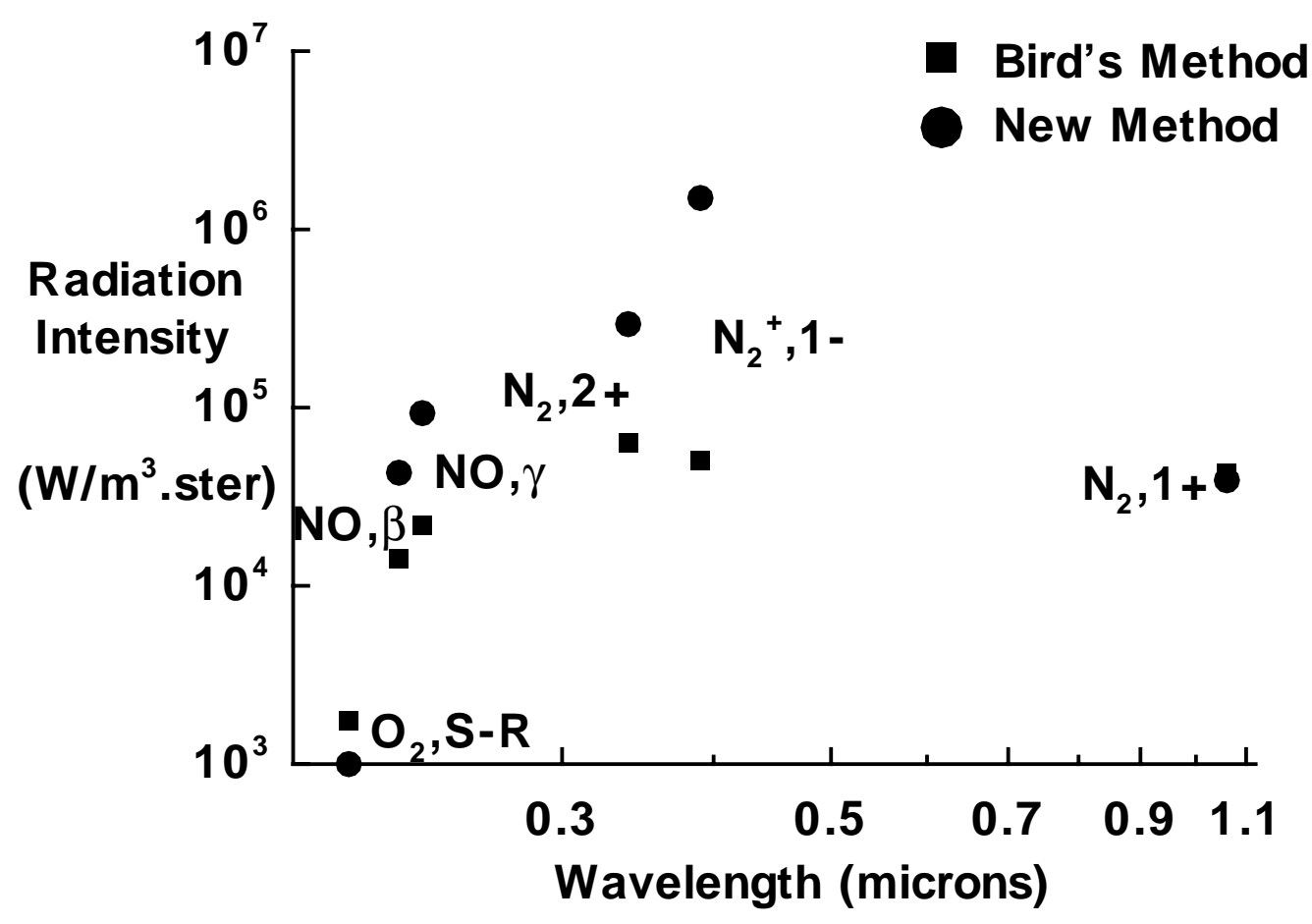


Fig. 10

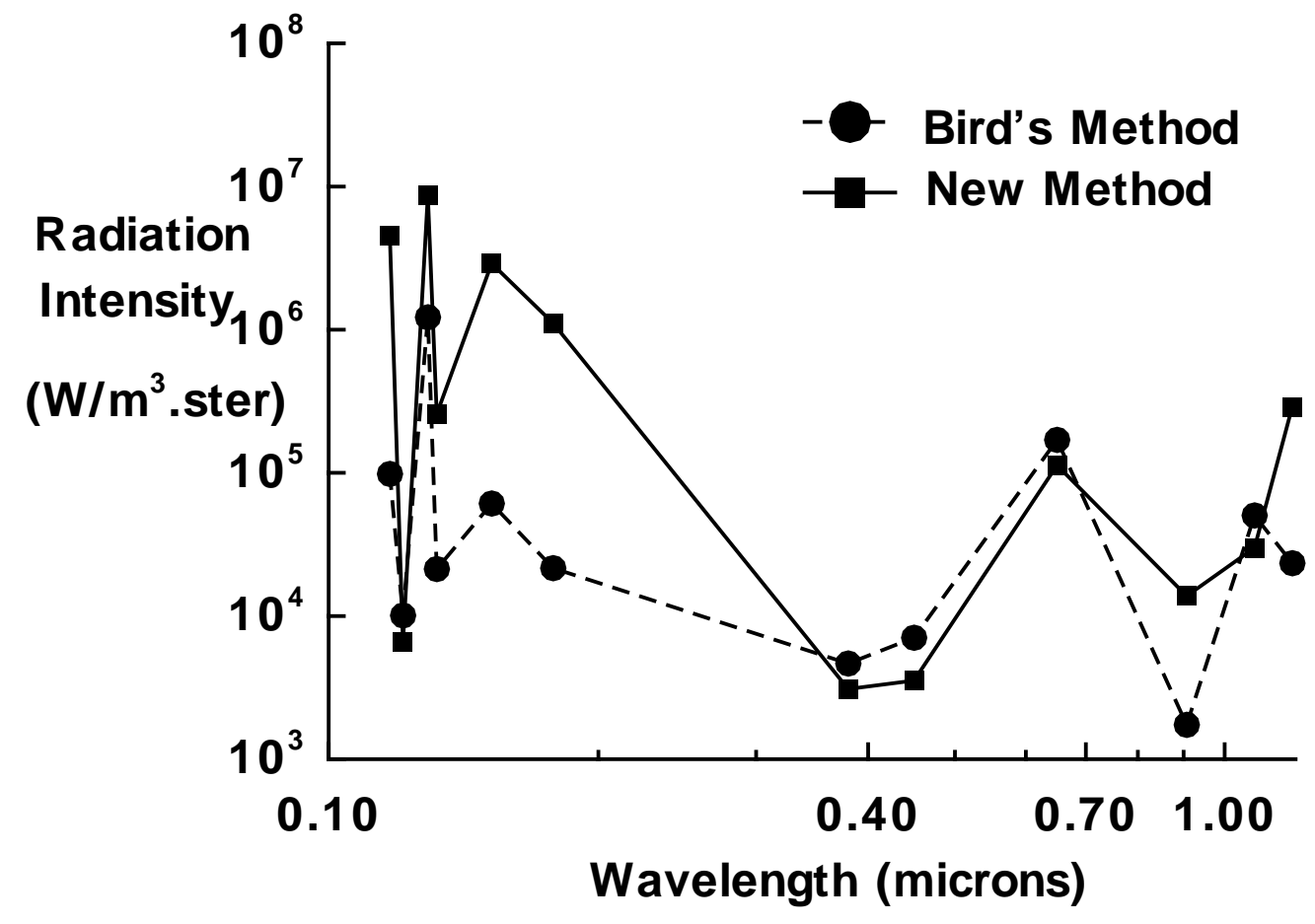


Fig. 11

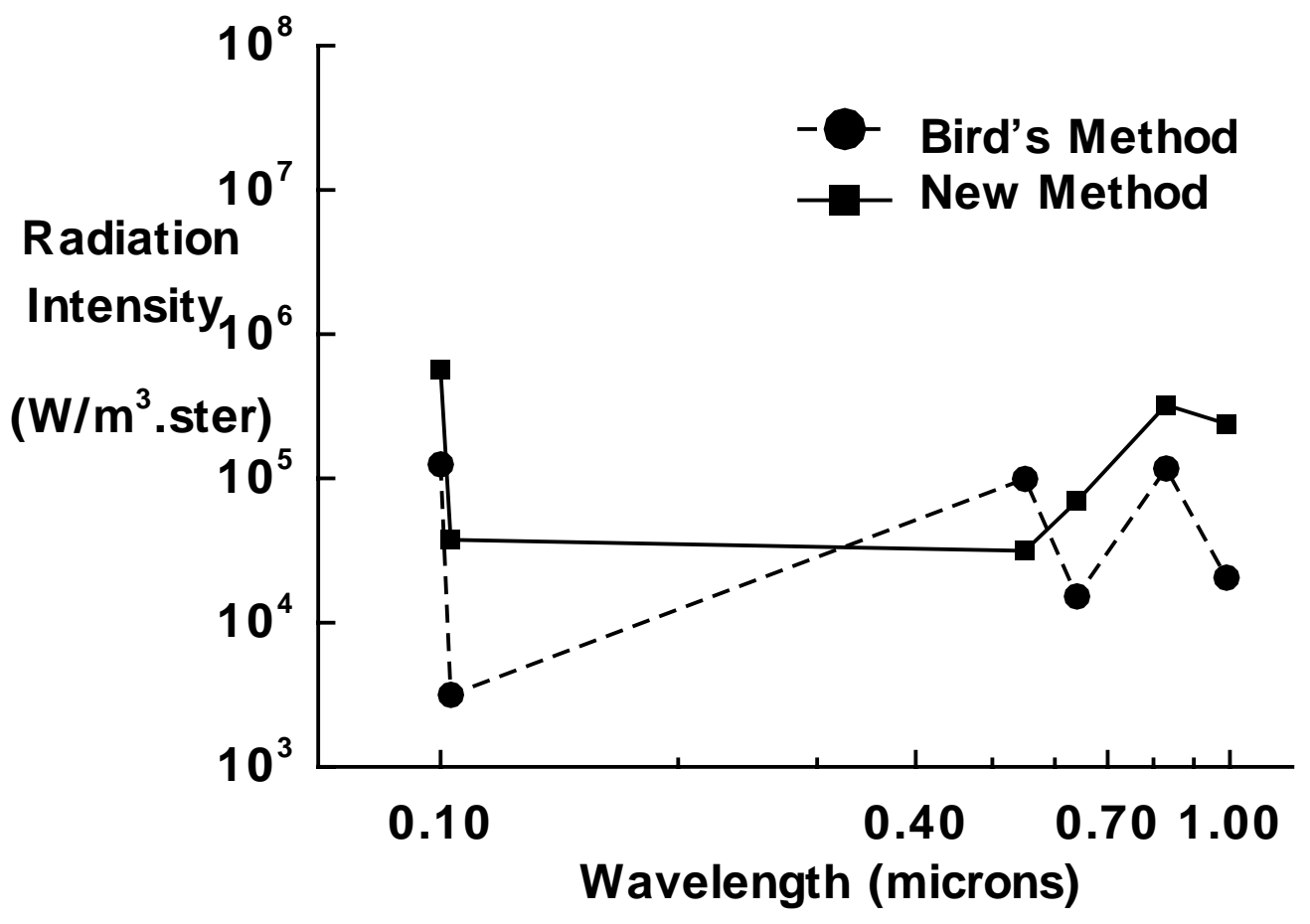

